\title{
La contribución del/de la auxiliar técnico educativo (ATE) para el desarrollo de una plena inclusión
}

\author{
Sebastià Verger Gelabert, Laura Chover, Maria Rosa Rosselló Ramón \\ Universidad Islas Baleares (España)
}

\section{RESUMEN}

Este estudio se centra en un grupo profesional hasta ahora ignorado: el/la Auxiliar Técnico Educativo. Mediante un diseño mixto que combina la aplicación del cuestionario SACIE-R y la entrevista en profundidad, en el que participaron 32 sujetos, se analiza el perfil y la labor que desarrolla este colectivo profesional, con el objetivo de describir y valorar qué función asume en la escuela, así como su contribución a la inclusión del alumnado con necesidades educativas especiales. Para el análisis de los datos cuantitativos se utilizó el programa Office Excel; en cambio, los datos cualitativos fueron tratados mediante el software Atlas.ti.

Los resultados avalan la conclusión que estamos ante un perfil profesional escasamente conocido, cuya tarea no siempre se ajusta a las demandas y realidades de las necesidades sociales del alumnado con discapacidad, lo que supone desaprovechar un valioso apoyo a la inclusión. Se incide en la necesidad de avanzar hacia la creación de equipos multidisciplinares que trabajen desde la cooperación para alcanzar metas educativas de mayor calidad. Las políticas inclusivas comunitarias y las iniciativas de innovación educativa deberán tener muy presente a este colectivo, ya que ejerce de verdadero puente entre el ámbito social y el educativo.

Palabras clave: Educación inclusiva, inclusión social, auxiliar técnico educativo, apoyo colaborativo

\section{The contribution of the education technical assistant (ATE) for the development of full inclusion}

\begin{abstract}
This study focuses on a professional group which has been ignored until now: education technical assistant. Through a mixed design that combines the application of the SACIE-R questionnaire and the in-depth interview, in which 32 subjects participated, the profile and the work carried out by this professional group are analyzed, with the aim of describing and assessing which function assumes in school, as well as its contribution to the inclusion of students with special educational needs. The Office Excel program was used to analyze the quantitative data; instead, qualitative data were processed using the Atlas.ti software.

The data collected support the conclusion that we are facing a poorly known professional profile, whose task does not always meet the demands and realities of the social needs of students with disabilities, which means missing valuable support for inclusion. The need to move towards the creation of multidisciplinary teams that work from cooperation to achieve higher quality educational goals is emphasized. Inclusive community policies and educational innovation initiatives should be very aware of this group, since it acts as a true bridge between the social and educational fields.
\end{abstract}

Key words: Inclusive education, social inclusion, educational technical assistant, collaborative support

ISSN: 0210-2773

DOI: https://doi.org/10.17811/rifie.49.2.2020.171-176 


\section{Introducción}

El acceso a una escolarización plena, capaz de combatir la exclusión social y la desigualdad entre los grupos más vulnerables, es uno de los grandes retos que plantea la sociedad actual (Programa Marco de la Unión Europea Horizonte 2020, H2020). Abordar este tema implica centrarse en el sistema educativo como un elemento decisivo para la compensación de desigualdades.

En poco tiempo, la educación inclusiva ha pasado de ser un discurso minoritario a estar cada vez más presente en la agenda política de los distintos países (OECD, 2015; UNESCO, 2015), dando pie a la apertura de una línea de investigación propia dentro del campo de la educación (Opertti, 2009; Cansino, 2017).

Bajo el lema de una Educación para Todos (UNESCO, 2000) se defiende, por una parte, que las diferencias entre las personas (por motivos de raza, sexo, idioma, religión, capacidades, orientación sexual, etc.) se acepten y se valoren positivamente (Blanco, 2008; Escudero y Martínez, 2012; Sapon-Shevin, 2013); por otra, que todo el alumnado se sienta incluido, es decir, reconocido y valorado (Ainscow, 2015). Para ello, se requiere reestructurar la institución escolar, promoviendo procesos de innovación y mejora que garanticen la presencia, la participación, el éxito y la autonomía de todo el alumnado, convirtiendo la diferencia en una concepción de vida (Ainscow, 2012).

Sin embargo, la generalización del modelo choca con muchos obstáculos y retrocesos hasta el punto que, diversos informes cuestionan su incidencia real en las aulas y centros escolares (UNESCO y UNICEF, 2015). Se trata de una tarea ardua, compleja, que incide de lleno en la esencia misma del propio sistema (Álvarez-Arregui y Arreguit, 2019). Como afirma Echeita (2006), la inclusión no tiene que ver únicamente con el alumnado, sino también con el personal docente y el resto de miembros de la comunidad educativa. Exige, entre otros factores, una apuesta clara por un modelo de apoyo colaborativo entre los profesionales y agentes implicados (Huguet, 2009; Rytivaara, 2012; Bair, 2013; Soldevila, Naranjo y Muntaner, 2017; Friend y Cook, 2017). Estos estudios avalan una redefinición del rol que asume el profesorado de apoyo de manera que se convierta en un recurso vinculado al aula, trabajando codo con codo con el profesorado tutor (Luque-Parra y Luque-Rojas, 2015), acompañando y posibilitando que cada niño desarrolle al máximo sus competencias y capacidades (Orozco y Moriña, 2019).

Compartiendo este marco teórico, esta investigación persigue dar un paso más, y dirigir el foco de atención hacia un tercer colectivo que trabaja en las escuelas, aunque sea catalogado como "personal no docente": el/la auxiliar técnico educativo (ATE). Pese a tener una creciente presencia en los centros escolares, la información y las investigaciones con respecto a este grupo profesional son todavía muy escasas.

Se trata de un colectivo que se caracteriza, en primer lugar, por situarse a caballo entre el personal docente y el personal de administración y servicios. Asume responsabilidades de carácter educativo derivadas de la relación directa que mantiene con el alumnado; pero, por lo que respecta al régimen laboral, se aproxima más al personal de servicios (Teixidó y Brucart, 2009). En segundo lugar, aunque no existe un marco legal único y común en todo el estado, tomando como referencia la normativa reguladora de la comunidad de las Islas Baleares, para acceder a esta profesión se exige sólo el Graduado en Educación Secundaria, el título de ciclo formativo de grado medio o título equivalente. Sin embargo, sus funciones son educativas además de asistenciales:

En relación al alumnado con NEE, prestan asistencia y formación en las actividades de la vida diaria que no pueda hacer por él mismo (...); colaboran en la atención y cuidado del alumnado dentro del aula, en los cambios de aulas o servicios de este alumnado y en la vigilancia en los recreos y salidas, siempre bajo la responsabilidad del personal docente. Asimismo, y siempre en ausencia del alumnado con NEE, llevan a cabo la tarea con el resto del alumnado (...) (BOIB núm. 70 de 14/05/2009, p. 22).

En tercer lugar, dado que el/la ATE presenta características diferentes y complementarias a los colectivos profesionales con quien comparte escenario (el profesorado tutor y el profesorado de apoyo), resulta imprescindible que se sienta parte integrante del equipo educativo (Booth y Ainscow, 2015). De hecho, para que su presencia sea efectiva y beneficiosa, los profesionales deberán coordinarse concienzudamente a fin de que su trabajo resulte efectivo, haya colaboración y se generen respuestas más inclusivas (Ainscow, 2012; Berategi y Orcasitas, 2015; Angelides, Constantinou y Leigh, 2009): "La relación entre los equipos y la colaboración es simple: un equipo eficaz es un grupo de trabajo colaborativo" (Friend y Cook, 2017, p.153).

Con todo, la experiencia que viven los auxiliares en las aulas es muy variable: en algunas ocasiones, se encuentran al margen del aprendizaje y se encargan exclusivamente del alumno con nee; en otras, se reparten el trabajo e, incluso, hay casos en los que se ha llegado a asumir la responsabilidad del aula. En este sentido, Gaintza, Arostegui, Berasategi, Ozerinjauregi, Darretxe, Orcasitas y Monzón (2015) reconocen el relevante papel que pueden jugar estos profesionales para conseguir normalizar el proceso de escolarización y facilitar la inclusión social del alumnado con nee.

Compartiendo este marco de referencia, el estudio se propone recoger la voz de este colectivo profesional para describir y analizar las funciones que cumple, valorando de qué modo su labor contribuye al desarrollo de la inclusión.

\section{Metodología}

\subsection{Objetivos}

De acuerdo con la finalidad del estudio, el trabajo de campo se propone un doble objetivo: Analizar el rol profesional de los Auxiliares Técnicos Educativos en los centros educativos y valorar su posible incidencia en el proceso de inclusión.

\subsection{Instrumentos y procedimiento}

Se diseña una investigación que combina el uso de dos instrumentos: una primera fase, de carácter exploratorio, en la que se aplica el cuestionario SACIE-R; a ello le sigue una segunda fase, en la que se utiliza la entrevista en profundidad. La unión de ambas técnicas nos permite dar la voz a los profesionales y conocer sus experiencias y opiniones (Susinos y Parrilla, 2008), a la vez que resulta eficaz para reconocer a las personas como sujetos activos y competentes, en la dirección que proponen Berasategi y Orcasitas (2015).

El cuestionario titulado The Sentiments, Attitudes and Concerns about Inclusive Education Revised (SACIE-R) (Forlin, Earle, Loreman y Sharma, 2011) mide, mediante 15 ítems, las percepciones del profesional sobre la educación inclusiva considerando su opinión acerca del alumnado con nee, la aceptación del alumnado con diferentes necesidades y sus concepciones sobre la implementación de la inclusión en la dinámica escolar cotidiana, entendiendo que estas categorías están directamente vinculadas a las competencias profesionales que desarrollan los/las ATE.

En la segunda fase se diseña una entrevista que consta de 21 preguntas, con una previsión para su desarrollo de una hora de 
duración. La entrevista recoge las siguientes variables relativas al perfil de los participantes: sexo, años de dedicación, formación recibida y situación laboral ( $\mathrm{n}^{\mathrm{o}}$ de centros, ratio, horas de dedicación). Por otra parte, las preguntas giran en torno a las siguientes dimensiones: formación, acogida y relaciones en el centro, roles, percepciones sobre las funciones del/de la ATE en relación a la inclusión educativa y social del alumnado con nee. Con todo, dado el carácter abierto de la entrevista, se incorporaron aquellas respuestas redundantes que surgieron a lo largo de la conversación mantenida con los participantes.

Para el análisis de los datos, las respuestas procedentes del cuestionario se volcaron en una hoja diseñada específicamente para ello, mediante el programa Office Excel. Se categorizaron y codificaron los diferentes ítems y se procedió a sumar los valores asignados a cada respuesta. En la segunda fase, el análisis de los datos cualitativos se llevó a cabo mediante el método de comparaciones constantes y la codificación abierta y axial de los datos, siguiendo las orientaciones de Glaser y Strauss (2017) usando para ello el software Atlas.ti. Tras varias lecturas de las transcripciones se realizó un primer acercamiento interpretativo y se identificaron los códigos apriorísticos. De igual modo, fueron apareciendo códigos emergentes no planteados en el inicio. Finalmente, tras la codificación se pasó a la fase interpretativa.

En el desarrollo de ambas fases, se tuvo presente el seguimiento de los principios éticos como la negociación, la colaboración (todas las personas que participaron tuvieron la oportunidad de influir en el desarrollo de la misma), la confidencialidad, la imparcialidad, la equidad o el compromiso con el conocimiento, quedando recogidos todos ellos en el consentimiento informado.

\subsection{Participantes}

El primer paso que se dio fue solicitar a la Consejería de Educación el listado de los/las ATE que durante el curso 2016-17 estaban trabajando en los centros escolares. Según este listado, el universo del estudio estaba compuesto por 114 profesionales. De forma aleatoria se mandó el cuestionario a una muestra formada por 40 profesionales, obteniendo una respuesta positiva por parte de 24 sujetos, lo que representa el $21 \%$ de la población.

Los rasgos que caracterizan esta primera muestra (Fase I) del estudio son los siguientes:

a) Puesto de trabajo: un 50\% trabaja en educación infantil; un $16,7 \%$ en educación primaria; un $25 \%$ en educación secundaria; $y$, un $8,3 \%$ en un centro de educación especial.

b) Sexo: un $87,5 \%$ es de sexo femenino y un $12,5 \%$ de sexo masculino.

c) Edad: un $66,7 \%$ cuenta con más de 46 años; un $25 \%$ tiene una edad comprendida entre 36 y 45 años; $y$, finalmente, la edad de $8,3 \%$ oscila entre los 26 y 35 años.

d) Años de experiencia en la profesión: un 54,2\% tiene de 6 a 15 años de experiencia; un 20,8\% tiene más de 16 años de experiencia; un $12,5 \%$, posee de 3 a 5 años; finalmente, un $12,5 \%$ posee 2 o menos años de experiencia.

Para la segunda fase se solicitó la participación voluntaria a todas aquellas profesionales de las que se obtuvo el contacto. Al final, para el desarrollo de las entrevistas se contó con la participación de 8 auxiliares: 4 ejercen en centros de Educación Infantil, 3 en Primaria y 1 en la etapa de secundaria.

\section{Resultados y discusión}

\subsection{Situación laboral}

Por lo que se refiere a la situación laboral, la mayoría de las participantes tienen jornadas laborales de $5 \mathrm{~h}$ y $22 \mathrm{~min}$ al día durante el curso escolar, lo cual implica que empiezan y acaban el mismo día que el alumnado y no cobran el resto del año. Este hecho, tiene múltiples consecuencias como recogen las entrevistas; por ejemplo, que el alumnado al que atienden no pueda acudir a actividades como el comedor, la escuela matinal o salidas extraescolares, pues aunque se requiere de la presencia del/de la ATE en todas ellas, queda fuera de su horario laboral.

La ratio de alumnado que atiende cada ATE es variable y se traduce en que algunas auxiliares atienden a 5 alumnos/as mientras que otras solo a 1 o 2 alumnos. Esto será relevante para valorar con cuántos docentes debe coordinarse el/la ATE y la diversidad de necesidades que debe atender.

\subsection{Relación con el personal docente}

La relación con el personal docente es muy variable, no obstante, tras el análisis de las entrevistas realizadas, se han encontrado puntos en los que sus situaciones confluyen:-por un lado, el que se refiere a la imagen tan arraigada que sigue habiendo del auxiliar como "cambia pañales", aspecto que comentan 5 de las entrevistadas. Por otro lado, han puesto de manifiesto cuánto les ha costado hacerse un lugar en el centro y cómo han tenido que demostrar, más que cualquier otro profesional, su valía.

Cuatro de las entrevistadas, señalan que las diferencias contractuales con el personal docente conllevan problemas de convivencia. Dos de las entrevistadas, por el contrario, manifiestan haberse sentido bien acogidas: "Sí que me he sentido acogida, respectada y integrada [...] siempre se ha dado un respeto infinito hacia el lugar que ocupa cada una de nosotras" (ATE2, líneas $24-25,42-43)$. En cualquier caso, destaca que el 52,2\% de las respuestas del cuestionario está de acuerdo o muy de acuerdo al considerar que las relaciones con el equipo docente son complejas o no ha resultado fácil hacerse un lugar en el centro.

\subsection{Formación}

La mayoría de sujetos entrevistados tienen estudios superiores a los exigidos para acceder al puesto de trabajo. Un $50 \%$ de la muestra ha cursado un Grado o estudios equivalentes; un 20,8\% tiene estudios secundarios; por último, el 29,2\% responde seleccionando el apartado otros estudios.

\subsection{Valoración de la formación exigida}

El 78,2\% manifiesta que le preocupa no tener los conocimientos ni habilidades requeridas para atender los alumnos con nee, mientras que un $21,8 \%$ declara tener un nivel alto. En general, los/las ATE consideran que la formación que se les exige es insuficiente. En este sentido, durante la entrevista dos de las participantes matizan que la formación exigida coincide con las funciones declaradas en la normativa y que, precisamente, es la normativa de acceso la que debería revisarse al no corresponderse con el modelo de escuela inclusiva: "Si no se revisan las funciones, sí. El problema está cuando nos planteamos si son estas funciones las que corresponden a un modelo de escuela inclusiva? Entonces, la respuesta es no" (ATE1, líneas 11-12). 


\subsection{Funciones y rol del/la ATE en el aula}

El $82,6 \%$ opina que las funciones no están bien definidas y no son conocidas por la comunidad educativa. El 95,5\% piensa que la función primordial del/la ATE consiste en normalizar el proceso de escolarización y superar las dificultades que conlleva la discapacidad.

Cuatro de las entrevistadas explican que el principal problema es que en las escuelas se desconocen sus funciones: "Es que no tienen ni idea de lo que es una ATE, de cuáles son mis funciones. La directora me dijo: por favor, me mandarás un e-mail o alguna cosa que recoja tus funciones?" (ATE5, líneas 74-76).

En este sentido, durante las entrevistas las auxiliares reconocen que desarrollan funciones que sobrepasan a las estipuladas en la normativa vigente. Mencionan tareas como adaptar las actividades del alumnado al que atienden, desarrollar la rutina de leer cuentos, organizar juegos en el patio o encargarse de trabajar los sistemas de comunicación alternativos. Cabe destacar que las mismas auxiliares apuntan que este tipo de actuaciones las hacen de manera voluntaria, pero las consideran fundamentales para la inclusión del alumnado.

Dos de las auxiliares participantes trabajan sentadas al lado de sus alumnos/as, en un caso porque la situación del alumno en cuestión lo requiere y, en otro, porque la tutora del aula no da otra opción, ni permite el desarrollo de otras funciones. El resto de las participantes trata de dar ayuda al discente aumentando progresivamente la distancia e interactuando con el resto del grupo para promover la autonomía y evitar la dependencia y el etiquetaje del alumnado con nee.

Otro dato que se extrae del análisis de las entrevistas es que la mitad de las auxiliares manifiestan realizar con el alumno/a al que atienden tareas diferentes al resto del grupo. En este punto cabe mencionar la situación de las/los Intérpretes de Lengua de Signos que son contratados bajo la misma categoría laboral que los/las ATE, lo que hace aún más diverso si cabe este perfil profesional.

Finalmente, un punto que ha surgido durante las entrevistas es el debate relacionado con la administración de medicamentos: aunque no está entre sus funciones, las auxiliares entrevistadas mantienen diversas posturas al respecto, que van desde la asunción de dicha responsabilidad hasta su completa negación.

\subsection{Coordinación con el resto de profesionales}

Cuando se aborda el tema de la coordinación con el resto de profesionales del centro el resultado que se obtiene es que solo 3 de las entrevistadas acuden a las reuniones de coordinación, 2 de las cuales desarrollan su actividad en la etapa de Educación Secundaria Obligatoria. La tercera trabaja en un aula UECCO (Unidad Educativa Específica en Centro Ordinario) de un centro de Educación Infantil y Primaria. Es importante destacar que las tres aseguran que su situación no es la común. Las 5 ATE restantes comentan que no acuden a ninguna reunión

\subsection{Situación emocional del/las ATE}

Tres de las entrevistadas manifiestan sentirse respetadas y valoradas; el resto, usa términos negativos para referirse a su situación: "frustrada" o "poco valorada y realizada". Asimismo, sienten que no se cuenta con su opinión en muchas ocasiones y que se sienten fuera de lugar.

Por último, una de las auxiliares explica en varias ocasiones de la entrevista que cree que el colectivo podría ofrecer más, siendo un recurso desaprovechado, limitado por aspectos rela- cionados con sus funciones, la falta de reconocimiento o el mismo horario.

\subsection{Relación del/la ATE con el alumnado y su familia}

Seis de las participantes manifiestan tener un vínculo afectivo muy fuerte y un conocimiento muy profundo del alumnado al que atienden, entre otros motivos, debido a la gran cantidad de horas que pasan con él. Aun así, las profesionales manifiestan su deseo de no crear una excesiva dependencia, aspecto que se ha conseguido al menos en 3 de los 8 casos analizados. Así mismo, manifiestan mantener buenas relaciones con las familias.

\subsection{Relación del alumnola con el resto de agentes: sus igua- les y docentes}

El 91,3\% opina que las relaciones del alumnado con nee con sus iguales son positivas. El 65,2\% piensa que las relaciones del docente con dicho alumnado son muy satisfactorias.

Tales resultados son matizados en las entrevistas, ya que en opinión de las auxiliares entrevistadas las relaciones del alumnado con nee con sus iguales son bastante dispares. Según las participantes en el estudio, los factores que influyen en la situación son: el tutor/a, el comportamiento del mismo alumno/a y el comportamiento del/de la ATE. Se dan situaciones en que nadie juega con dicho alumnado; otras más específicas en las que el alumno/a tiene relación con iguales del sexo opuesto, mientras que es rechazado por los compañeros de su mismo sexo; y finalmente, otras en que sus iguales le ayudan y le quieren.

En segundo lugar, se describe una situación bastante crítica por lo que se refiere a la relación del alumnado con nee con el profesorado tutor. La mayoría de tutores/as tienden a pensar que dicho alumnado es responsabilidad del auxiliar e interactúa poco en él: "la relación en el aula así como mi actuación depende mucho de cada tutor. A veces, les cuesta sentir que los alumnos son sus alumnos y sienten que es responsabilidad de la ATE" (ATE2, líneas 191-193).

\subsection{Inclusión}

El 95,7\% manifiesta su preocupación ante la dificultad para ofrecer una atención adecuada a todos los estudiantes en una aula inclusiva. Sobre este punto del análisis de las entrevistas se han recogido las siguientes afirmaciones:

Las 2 ATE que trabajan en las aulas UECCO, explican que estos alumnos acuden al aula ordinaria en asignaturas como Plástica o Música y, generalmente, hacen actividades ligeramente diferentes al resto.

El resto de las entrevistadas consideran que su presencia en el aula junto al alumnado con nee fortalece su etiquetaje, por lo que siempre que es posible incrementan progresivamente la distancia e interactúan con otros compañeros/as.

Asimismo, hay unanimidad con respecto a la idea de que el profesorado tutor es un elemento clave en la inclusión, puesto que cuando acepta al alumnado con nee, el resto del alumnado también lo hace. Tres de las participantes afirman que el alumno/a al que atienden está encasillado y excluido del grupo, mientras que el resto manifiesta una opinión en sentido opuesto.

\section{Demandas y sugerencias}

Entre las propuestas de mejora, las participantes destacan el deseo de formar parte del equipo docente y que, entre sus obligaciones, esté la de acudir a las reuniones para que se incremente la 
comunicación y coordinación con el resto de profesionales. Para ello, en su opinión, sería fundamental ampliar su horario y dejar de ser personal laboral. Otra de las propuestas sugeridas hace referencia a que haya una mayor oferta de formación continua y de que se incluya al colectivo en la formación de cada centro, así como que se cree una propuesta formativa específica e incluida en la oferta regulada, como podría ser una formación profesional de Auxiliar Técnico Educativo, a semejanza del título de Auxiliar Técnico Sanitario: "si pudiera cambiaria la base, o sea, que se estudie, que se haga un módulo, o lo que sea, y formar a la gente específicamente para esto" (ATE8, líneas 286-288). También, se menciona la voluntad de que su trabajo se vea compensado con un sueldo digno.

En resumen, como se refleja en la gráfica 1, los datos indican que estamos ante un colectivo profesional que posee más formación de la exigida, cuyas funciones son desconocidas en la comunidad educativa, hecho que se acompaña con una baja percepción de dominio de sus conocimientos profesionales.

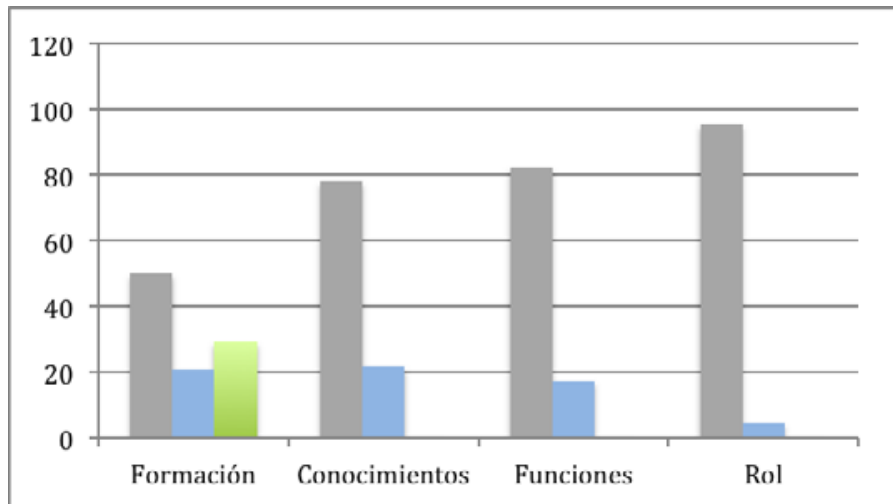

Gráfica 1. Resumen de los principales indicadores cuantitativos.

\section{Conclusiones}

Buena parte de las participantes en la investigación tienen un nivel de estudios superiores al que marca la normativa que rige el acceso al puesto de trabajo. A pesar de ello, un 78,2\% manifiesta una cierta preocupación por los escasos conocimientos y habilidades que posee para atender al alumnado con nee, especialmente desde el modelo de escuela inclusiva. A ello se añade el hecho de que este colectivo profesional es contratado como personal laboral y está sometido a unas condiciones laborables variables y restrictivas, con ratios que oscilan entre 5 y 2 alumnos, que no permiten atender todas las necesidades que presenta en su quehacer diario dicho alumnado.

Las funciones de los/las ATE son difusas, ambiguas y poco conocidas, lo que se convierte en un punto conflictivo en los centros educativos. Estos resultados coinciden con los datos apuntados por Texidó y Brucart (2009), quienes recogen los dilemas que provoca esta dualidad de funciones: “¿Le ayudará a sonarse, pero, en cambio, no le orientará a la hora de coger bien las tijeras? ¿Le ayudará a reconocer y mencionar los errores en un ejercicio? ¿Ignorará a otro alumno que le pida ayuda?" (p.61).

Por otra parte, las auxiliares reconocen que desarrollan funciones que sobrepasan a las estipuladas en la normativa vigente aunque ello queda a merced de cada auxiliar y del modelo didáctico que asume el profesorado tutor. Este hecho dificulta encontrar un lugar en el aula, así como poder mantener unas relaciones fluidas con el profesorado. Continua existiendo la etiqueta de cambia pañales, función prioritaria en una escuela que continua anclada bajo el paraguas de la integración escolar.
Con contadas excepciones, los/las ATE no acuden a las reuniones de coordinación, ni a la formación que realiza el profesorado del centro. Este hecho junto con la indefinición de las tareas que debe realizar en el aula, genera una situación emocional que es vivida por este colectivo profesional desde el prisma negativo. La mayoría usa los calificativos de poco valoradas, escasamente reconocidas... manifestando que no se cuenta con su opinión en muchas ocasiones y que se sienten fuera de lugar

Las participantes manifiestan tener una relación positiva, sólida y profunda con el alumnado al que atienden, así como con sus familias. Así pues, son una fuente de información muy valiosa a la que convendría dar espacio y voz. Los/las ATE son un recurso humano más dentro del aula, con un tremendo potencial que, aprovechado de manera óptima, puede beneficiar a todos y todas, incluido el mismo profesorado. Tal y como sugieren autores como Ainscow (2012) o Gallego (2011), los apoyos existentes en los centros educativos deben ser para todos y todas y nunca exclusivos, pues, aunque puedan ser imprescindibles para algunos, benefician altamente al resto.

No ocurre lo mismo cuando opinan sobre la relación que mantiene el alumnado con nee con sus compañeros de clase o con el profesorado tutor, detectando situaciones bastante críticas tanto a nivel social como académico, situaciones que, como mínimo, son mejorables desde el modelo de educación inclusiva. Para los/las ATE su rol en relación al alumnado y la actitud del tutor son dos elementos determinantes para conseguir una verdadera inclusión. Desde nuestro punto de vista, la inclusión exige ir más allá de la situación académica, ya que no puede desarrollarse adecuadamente con independencia de considerar el grado de inclusión social y los alcances de las brechas de equidad en la adquisición de competencias y de aprendizajes.

Con todo, los datos dibujan un perfil profesional que permanece anclado a una visión educativa más cercana a la integración que a la inclusión, donde el/la ATE realiza funciones de cuidado físico del alumnado con nee, trabajando en solitario y/o con escasa conexión con el profesorado, pero con una gran capacidad para observar y recopilar una información altamente valiosa en el desarrollo educativo y social del alumnado. En la línea de propuestas presentadas por Irazabal, Pastor y Molina (2016), pensamos que la figura profesional del/de la ATE podría ser un recurso clave para la inclusión de las personas con nee desde la infancia, ejerciendo de puente entre la familia y los diversos contextos sociales donde se desarrolla la persona afectada. En este sentido sería importante atender la demanda que realizan estas profesionales al exigir que se les ofrezca más formación y un rol más activo y participativo para dar una mejor respuesta a las demandas que se presentan en los centros educativos.

En resumen, tal como se desprende de los datos anteriores, para avanzar hacia horizontes más inclusivos es urgente repensar las funciones y tareas que asume este colectivo profesional, otorgándole un carácter más inclusivo, lo que supone dejar de concebirlo como un recurso exclusivo del alumnado con nee, para incorporarlo activamente en las estructuras de apoyo, en la dirección propuesta por Soldevila et al. (2017). De ello se deriva la necesidad de avanzar hacia la creación de equipos multidisciplinares que trabajan desde la cooperación para alcanzar metas educativas de mayor calidad. Por eso, las políticas comunitarias y las iniciativas de innovación educativa deberán tener muy presente a este colectivo, si quieren continuar apostando por una concepción comunitaria de la infancia y una mayor protección de los colectivos vulnerables.

Finalmente se constatan algunas de las limitaciones que pre- 
senta el trabajo de campo. Entre estas, cabe destacar, dada la pluralidad de factores que intervienen y las situaciones tan dispares existentes dentro del mismo colectivo, la necesidad de ampliar la muestra del estudio, tanto desde el punto de vista cuantitativo, para obtener una muestra estadísticamente representativa, como en la parte cualitativa, recogiendo la voz de otros protagonistas implicados (equipos directivos, profesorado tutor y familias). La otra limitación ha radicado en la cantidad de auxiliares entrevistadas, aspecto a tener en cuenta en investigaciones futuras.

\section{Referencias bibliográficas}

Ainscow, M. (2012). Haciendo que las escuelas sean más inclusivas: lecciones a partir del análisis de la investigación internacional. Revista Educación Inclusiva, 5 (1), 39-49.

Ainscow, M. (2015). The Index for Inclusion. En Struggles for Equity in Education (pp. 95-105). Routledge. doi: 10.3102/0091732X03000106

Álvarez-Arregui, E. y Arreguit, X. (2019). El futuro de la Universidad y la Universidad del futuro. Aula Abierta, 48, 4, 447- 479 doi: https: / / doi.org/1017811/rifie.48.4.2019.349-372

Angelides, P., Constantinou, C. y Leigh, J. (2009). The role of paraprofessionals in developing inclusive education in Cyprus. European Journal of Special Needs Education, 24 (1), 7589. doi: https://doi.org/10.1080/08856250802596741

Bair, M. A. (2013). Restricted professionalism of teachers: Implications for collaboration. En S. Conley and B. Cooper (Eds.), Moving from teacher isolation to collaboration (pp. 17-34). Lanham, Maryland: Rowman y Littlefield Education.

Berasategi, N. y Orcasitas, J.R. (2015). Buenas prácticas inclusivas: desde la escolarización de alumnado con EERR hacia la Escuela Inclusiva para toda la ciudadanía. En Z. Gaintza, I. Arostegui, N. Berasategi, N. Ozerinjauregi, L. Darretxe, J.R. Orcasitas, y J. Monzón (Eds.). La innovación escolar desde la perspectiva de personas con enfermedades raras en el País Vasco: historias de vida, prácticas escolares, necesidades del sistema educativo y propuestas de mejora para una escuela y sociedad inclusiva (pp. 111-138). Bilbao: FEDER Euskadi.

Blanco, R. (2008). Marco conceptual sobre educación inclusiva. En R. Blanco, I. Aguerrondo, A. Ouane y S. Shaeffer (Eds.). La educación inclusiva: el camino hacia el futuro (pp. 5-14.). Ginebra: Organización de las Naciones Unidas para la Educación, la Ciencia y la Cultura.

Resolución del director general de Trabajo mediante la cual se dispone el registro y la publicación oficial del Acuerdo de la Comisión Negociadora del Convenio Colectivo del personal laboral de la Comunidad Autónoma de las Islas Baleares. (14 de mayo de 2009). Boletín Oficial de las Islas Baleares (BOIB), número 70. Palma de Mallorca-Gobiernos de las Islas Baleares.

Booth, T. y Ainscow, M. (2015). Index for inclusion: developing learning and participation in schools (3r Ed.). Bristol: CSIE.

Cansino, P. A. P. (2017). Inclusión educativa y cultura inclusiva. Revista de Educación Inclusiva, 10(2), 213-226.

Echeita, G. (2006). Educación para la inclusión o educación sin exclusiones. Madrid: Narcea.

Escudero, J.M. y Martínez, B. (2012). Las políticas de lucha contra el fracaso escolar: ¿programas especiales o cambios profundos del sistema y la educación? Revista de Educación, número extraordinario, 174-193. doi: 10.4438/1988-592X-RE-2012EXT-211

Forlin, C., Earle, C., Loreman, T. y Sharma, U. (2011). The Sentiments, Attitudes and Concerns about Inclusive Education Revised (sacie-r). Scale for Measuring Pre-Service Teachers'
Perceptions about Inclusion. Exceptionality Education International, 21 (1), 50-65.

Friend, M. y Cook, L. (2017). Interactions: Collaboration skills for school professionals. (8th ed.). Boston, MA: Pearson Education Inc.

Gaintza, Z., Arostegui, I., Berasategi, N., Ozerinjauregi, N., Darretxe, L., Orcasitas, J.R. y Monzón, J. (2015). La innovación escolar desde la perspectiva de personas con enfermedades raras en el País Vasco: historias de vida, prácticas escolares, necesidades del sistema educativo y propuestas de mejora para una escuela y sociedad inclusiva. Bilbao: FEDER Euskadi.

Gallego, C. (2011). El apoyo inclusivo desde la perspectiva comunitaria. Revista Interuniversitaria de Formación del profesorado, (70), 93-110.

Glaser, B. G. \& Strauss, A. L. (2017). Discovery of grounded theory: Strategies for qualitative research. New York: Routledge.

Huguet, T. (2009). El trabajo colaborativo entre el profesorado como estrategia para la inclusión. En C. Giné (Coord.), La educación inclusiva. De la exclusión a la plena participación de todo el alumnado (pp.81-94). Barcelona: ICE, Universitat de Barcelona.

Irazabal, M., Pastor, C. y Molina, M.C. (2016). Family impact of care and respite service: life experiences of mothers of adult children with intellectual disability and mental disorders. Revista de cercetare si interventie sociala, 55, 7-18.

Luque-Parra, D.J. y Luque-Rojas, M.J. (2015). Alumnado con necesidades específicas de apoyo educativo: aspectos psicopedagógicos en un marco inclusivo. Perspectiva Educacional, 54 (2), 59-73. doi: 10.4151/07189729-Vol.54-Iss.2-Art.333.

OECD (2015). Education Policy Outlook 2015: Making reforms happen. París: OECD Publishing. doi: http://dx.doi.org/10.1787/9789264225442-en

Opertti, R. (2009). La Educación Inclusiva, perspectiva internacional y retos de futuro. El largo camino hacia una educación inclusiva. Aportaciones desde la historia de la educación, 117-138.

Orozco, I. y Moriña, A. (2019). Prácticas Docentes para una Pedagogía Inclusiva en Educación Primaria: Escuchando las voces del Profesorado. Aula abierta, 48, 3, 331-338. doi: https:/ /doi. org/10.17811/rifie.48.3.2019.331-338.

Programa Marco de la Unión Europea Horizonte 2020, H2020. Recuperado de https:/ / ec.europa.eu/programmes/horizon2020/ sites/horizon2020/files/H2020_ES_KI0213413ESN.pdf doi: $10.2777 / 80075$

Rytivaara, A. (2012). Collaborative classroom management in a co-taught primary school classroom. International Journal of Educational Research 53, 182-191. doi: 10.1016/j.ijer.2012.03.008

Sapon-Shevin, M. (2013). La inclusión real: una perspectiva de justicia social. Revista de Investigación en Educación, 3 (11), 71-85.

Soldevila, J., Naranjo, M. N. y Muntaner, J. J. M. (2017). Inclusive practices: the role of the support teacher. Aula abierta, 46, 4956. doi: https://doi.org/10.17811/rifie.46.2017.49-56

Susinos, T. y Parilla, A. (2008). Dar la voz en la investigación inclusiva. Debates sobre inclusión y exclusión desde un enfoque biográfico-narrativo. REICE. Revista Iberoamericana sobre Calidad, Eficacia y Cambio en Educación, 6 (2), 157-171.

Teixidó, J. y Brucart, R. (2009). Perfils professionals emergents a l'escola: una mirada organitzativa. Guix: elements d'acció educativa, (355), 57-64.

UNESCO (2000). Marco de acción de Dakar. Educacion para Todos: cumplir nuestros compromisos comunes. París: UNESCO.

UNESCO Institute for Statistics y UNICEF (2015). Fixing the broken promise of education for all. Finding from the Global Initiative on Out-of-School Children. Montreal: UIS. doi: http:/ / dx.doi.org/10.15220/978-92-9189-162-7-en 